intensity of TGC therapy. Treatments with biologics and systemic glucocorticoids, but not with MTX, were associated with lower degree of inflammation in AC.

Conclusion The results showed that the application of systemic biological therapy may result in less intensive TGC therapy, resulting in glucocorticoid-sparing potential, and reducing intraocular inflammation.

\section{DETERMINING THE EFFECTIVENESS OF SYSTEMIC IMMUNOMODULATORY THERAPY IN THE TREATMENT OF PATIENTS WITH JUVENILE IDIOPATHIC ARTHRITIS ASSOCIATED UVEITIS DEPENDING ON THE CHOSEN OUTCOME MEASURES}

${ }^{1}$ Marija Barisic Kutija ${ }^{2}$ Mario Sestan* ${ }^{1}$ Sanja Peric ${ }^{2}$ Nastasia Kifer ${ }^{1}$ Petra Kristina Ivkic, ${ }^{1}$ Martina Galiot Delic, ${ }^{1}$ Josip Knezevic, ${ }^{2}$ Martina Held, ${ }^{2}$ Marijan Frkovic, ${ }^{2}$ Marija Jelusic ${ }^{1}$ Nenad Vukojevic. 'University Hospital Centre Zagreb, Department of Ophthalmology, University of Zagreb School of Medicine; ' University Hospital Centre Zagreb, Department of Pediatrics, University of Zagreb School of Medicine, Zagreb, Croatia

\subsection{6/archdischild-2021-europaediatrics.453}

Indroduction The number of patients with juvenile idiopathic arthritis associated uveitis (JIA-U) who require systemic immunomodulatory treatment (IMT) for disease control is small. Variabilities in the patient selection and the results on the effectiveness of IMT make it difficult to compare studies. We aimed to show the difference between obtained levels of therapy efficacy on the same sample of JIA-U patients depending on the selected outcomes.

Methods The longitudinal observational study with JIA-U patients treated with IMT was conducted at University Hospital Centre Zagreb in the period from 2011 to 2017. We included 38 JIA-U patients aged 2 to 15 years and 69 eyes (7 patients had unilateral JIA-U).

Results At baseline 46 (66.7\%) eyes had grade $\leq 1+$ of nflammation in anterior chamber (AC) according to Standardization of Uveitis Nomenclature (SUN) Working Group criteria, 11 $(15.9 \%)$ had grade $2+, 3(4.4 \%)$ had grade $3+.23$ children $(60.5 \%)$ had already received methotrexate (MTX), 8 (21.0\%) biologics, $4(10.5 \%)$ systemic glucocorticoids (GC). Topical glucocorticoids (TGC) in the form of drops were used in 61 $(88.4 \%)$ eyes with a median of 4 daily doses, GC ointment in $75.4 \%$ of the eyes with a median of 1 dose. Until the end of the follow-up, all children received MTX, and 40\% of them biologics. The results of the effectiveness of IMT are presented according to the reduction of the need for TGC and achieved level of inflammation in AC. In the first 12 months, among JIA-U patients treated with both biologics and MTX, in $65 \%$ of eyes there was no need for TGC therapy, in the 48 th month in $50 \%$ of eyes, while the rest required 1-2 daily doses of TGC. At the end of the first year, with MTX and biological therapy $75 \%$ of eyes had grade 0 of inflammation and in 48 th month $61.1 \%$. In the 12 th month in $75 \%$ of eyes a grade 0 of inflammation was achieved with $\leq 2$ doses of TGC, and in the 48th month in $61.1 \%$. According to milder criteria, in the 12th month $90 \%$ of the eyes have a degree of inflammation $\leq 0,5+$ with $\leq 2$ doses of TGC, and in the 48th month all patients achieved this goal.

Conclusion It was shown that the results of treatment outcomes during follow-up largely depend on the selected outcome measures. This will be important for future research because setting different limits can lead to a more favorable outcome.

\section{DIFFERENT HISTOLOGICAL CLASSIFICATIONS FOR IGA VASCULITIS NEPHRITIS - WHICH ONE HAS THE BEST ASSOCIATION WITH THE DISEASE OUTCOME?}

${ }^{1} \mathrm{~N}$ Kifer*, ${ }^{1} \mathrm{M}$ Sestan, ${ }^{1} \mathrm{M}$ Held, ${ }^{1} \mathrm{M}$ Coric, ${ }^{1} \mathrm{~S}$ Bulimbasic, ${ }^{2} \mathrm{~T}$ Giani, ${ }^{3} \mathrm{~N}$ Martin, ${ }^{4} \mathrm{~S}$ Srsen, ${ }^{5} \mathrm{~A}$ Gudelj Gracanin, ${ }^{6} \mathrm{D}$ Kifer, ${ }^{7} \mathrm{M}$ Heshin, ${ }^{8} \mathrm{~A}$ Ravelli, ${ }^{9} \mathrm{R}$ Cimaz, ${ }^{10} \mathrm{~S}$ Ozen, ${ }^{11} \mathrm{~A}$ Gagro, ${ }^{1} \mathrm{M}$ Frkovic, ${ }^{1} \mathrm{M}$ Jelusic, on behalf of the PReS Vasculitis Working Party. 'University Hospital Centre Zagreb, University of Zagreb School of Medicine, Zagreb, Croatia; ${ }^{2}$ Anna Meyer Children's Hospital, Florence, Italy; ${ }^{3}$ Royal Hospital for Children, Glasgow, UK; ${ }^{4}$ University Hospital Centre Splt, University of Split School of Medicine, Split; ${ }^{5}$ University Hospital Dubrava, University of Zagreb School of Medicine, Zagreb, Croatia; ${ }^{6}$ Faculty of Pharmacy and Biochemistry, Zagreb, Croatia; 'Dana Dwek Children's Hospital, Tel Aviv, Israel; ${ }^{8}$ Division of Rheumatology, Giannina Gaslini Institute, Genoa; ${ }^{9}$ Division of Clinical Rheumatology, Research Center for Adult and Pediatric Rheumatic Diseases, Università degli Studi di Milano, Milan, Italy; ${ }^{10}$ Department of Pediatric Rheumatology, Hacettepe University, Ankara, Turkey; ${ }^{11}$ Children's Hospital Zagreb, Josip Juraj Strossmayer University of Osijek Medical Faculty Osijek, Zagreb, Croatia

\subsection{6/archdischild-2021-europaediatrics.454}

Introduction IgA vasculitis nephritis (IgAVN) is almost the only cause of morbidity and mortality among children suffering from this most common childhood-vasculitis. Several histological classifications are used in the analysis of renal biopsy findings in IgAVN, but it remains unknown which one is the best predictor of severity and disease outcome.

Objectives The aim was to compare the four most commonly used histologic classifications for IgAVN and to establish which variables of each histological classification have the strongest association with unfavorable outcome.

Methods The cross-sectional study included 69 patients with IgAVN (diagnosed by EULAR/PRES/PRINTO criteria) and available renal biopsy specimens for analysis using the four histological classifications for IgAVN (the International Study of Kidney Disease in Children (ISKDC) classification, the Oxford classification, the Haas histologic classification of IgA nephropathy and the modified semi-quantitative classification (SQC), developed by Koskela et al.). The clinical outcome was defined through four categories, graded according to the modified classification of Counahan (physical examination, hematuria, proteinuria, urine albumin-to-creatinine ratio, hypertension and eGFR). The linear relationships between outcome and histological classifications were analysed using ordinal regressions using the first-order of polynomial orthogonal contrasts.

Results The SQC classification proved to be the best, reducing the deviation (of the model-predicted outcome value from the observed value) by $9.5 \%(\chi 21=13,89, \mathrm{p}<0,001)$, followed by the Oxford classification with a deviation reduction of $8.0 \%$ $(\chi 21=11,76, \mathrm{p}=0,001)$, then the ISKDC classification with a decrease in deviation of $3.3 \% \quad(\chi 21=4,89, p=0,027)$. The worst was the Haas classification with a decrease in deviation of $2.1 \%(\chi 21=3,06, p=0,080)$. Analysis of individual variables of Oxford and SQC classifications showed that with increasing values in the variables of interstitial fibrosis ( $t 66=3,23$, $\mathrm{p}=0,002)$, tubular atrophy $(\mathrm{t} 66=2,94, \mathrm{p}=0,005)$ and tubular dilatation ( $t 66=2,40, p=0,019)$ in the SQC classification, and endocapillary hypercellularity $(\mathrm{t} 66=3,14, \mathrm{p}=0,003)$ and crescents $(\mathrm{t} 66=2,07, \mathrm{p}=0,043)$ in the Oxford classification the outcome worsens.

Conclusion This study showed that the SQC classification has the strongest association with the IgAVN severity and outcome. Although crescents on renal biopsy were considered the most important outcome indicators, our study suggests that tubulointerstitial changes could be more important as predictors of poor outcome. Interstitial and renal tubules changes 
should be further explored in order to have better predictive values of IgAVN outcome and to be incorporated into existing or new classifications, on the basis of which guidelines for the treatment of patients would be developed.

SUPPORT: Croatian Science Foundation project IP-2019-048822.

\section{SINGLE NUCLEOTIDE POLYMORPHISMS OF GENES HMGB1 AND AGER AND ITS ASSOCIATION WITH CLINICAL FEATURES OF IGA VASCULITIS}

\begin{abstract}
${ }^{1}$ Martina Held*, ${ }^{2}$ Mateja Batnozić Varga, ${ }^{1}$ Mario Sestan, ${ }^{2}$ Matej Sapina, ${ }^{1}$ Nastasia Kifer, ${ }^{1}$ Danica Grguric, ${ }^{1}$ Kristina Crkvenac Gornik, ${ }^{1}$ Marijan Frkovic, ${ }^{2}$ Nena Arvaj, ${ }^{2}$ Jasenka Wagner, 'Marija Jelusic. 'University Hospital Centre Zagreb, Department of Pediatrics, University of Zagreb School of Medicine; ${ }^{2}$ University Hospital Centre Osijek, Josip Juraj Strossmayer University of Osijek, Medical Faculty Osijek, Croatia
\end{abstract}

\subsection{6/archdischild-2021-europaediatrics.455}

Introduction IgA vasculitis (IgAV) is a disease in which genetic background also plays an important role. Some small studies have indicated the importance of variants in various non-HLA genes in the manifestation of different disease phenotypes.

Objectives The aim of this research was to investigate single nucleotide polymorphisms (SNPs) of genes HMGB1 and AGER encoding for high mobility group box-1 (HMGB1) and receptor for advanced glycation endproducts (RAGE), both acting as mediators of inflammation, in the susceptibility and clinical features of patients with IgAV.

Methods HMGB1 and RAGE gene polymorphisms were genotyped using a real-time polymerase chain reaction. The presence and frequency of polymorphisms in HMGB1 (rs2249825, rs1045411, rs1060348, rs1412125 and rs41369348) and RAGE (rs1800625, rs1800624, rs2070600 and rs3134940) were determined. Clinical data were collected from database of IgAV patients from two Croatian University Centers for pediatric rheumatology.

Results 81 pediatric IgAV patients were included, of whom 45 were boys and 36 girls, as well as 150 age- and sexmatched healthy controls without any history of autoimmune disease. The median (range) age of IgAV patients was 6.25 (4.60-8.20) years, and among them $71.6 \%$ had joint involvement, $29.62 \%$ had gastrointestinal manifestations, while $27.16 \%$ developed nephritis. The purpuric rash which extended from lower extremities to the trunk, upper extremities and face (generalized rash) was present in $43.20 \%$ of patients and $27.16 \%$ had at least one relapse. Among the analyzed polymorphisms, only in the rs1412125 there was a deviation from the Hardy Weinberger equilibrium. There was no statistically significant association of the analyzed polymorphisms with the IgAV susceptibility, compared to healthy controls. Polymorphism rs2070600 was significantly related with the development of nephritis in IgAV, while rs1412125 was associated with gastrointestinal involvement. The IgAV patients carrying the $\mathrm{T}$ allele (rs2070600) of the AGER had significantly increased risk of nephritis development compared with the IgAV patients with homozygous CC genotype in dominant (OR 4.05, CI 1.09-15.03, p = 0.037) and additive genetic models (OR 3.95, CI 1.16-13.47, $\mathrm{p}=0.049$ ). The minor C allele (rs1412125) of the HMGB1 was found to significantly increase the risk of gastrointestinal involvement in overdominant model with an allelic odd ratio of 2.78 (CI 1.04-7.43, $\mathrm{p}=0.04$ ).
Conclusion Although neither of analyzed HMGB1 and RAGE polymorphisms was not associated with IgAV susceptibility, our results indicated that these polymorphisms may be involved in the pathogenesis of IgAV with possible effect on different disease phenotypes.

SUPPORT: Croatian Science Foundation project IP-2019-048822 .

\section{M2 MACROPHAGE INFILTRATION AND HENOCH- SCHÖNLEIN'S PURPURA NEPHRITIS}

Nastasia Kifer*, Mario Sestan, Martina Held, Domagoj Kifer, Marijan Frkovic, Emina Babarovic, Stela Bulimbasic, Marijana Coric, Alenka Gagro, Gordana Laskarin, Marija Jelusic. University Hospital Centre Zagreb, Department of Paediatrics, University of Zagreb School of Medicine

\subsection{6/archdischild-2021-europaediatrics.456}

Goal Having in mind that macrophages frequently infiltrate injured glomerular and tubulointerstitial tissue, it is possible that the degree and subtype of macrophage infiltration varies depending on the type and severity of renal injury. The aim of this research was to determine macrophage subclasses in renal biopsy specimens of HSPN patients and to analyze their quantity in regard with patients ${ }^{6}$ clinical parameters and histologic features.

Method We performed an immunohistochemical study on renal tissue samples of patients with HSPN, diagnosed by EULAR/PRINTO/PRES criteria and followed for at least 6 months. Patient clinical and laboratory data was retrieved from hospitals' medical records. Renal biopsy samples were marked with antibodies for CD68, iNOS and arginase. The number of immunoreactive cells was counted in each glomerulus by two independent experts.

Results Laboratory and histologic data for 25 patients with HSPN was evaluated in regard with macrophage infiltration of the renal tissue. The median glomerular $\mathrm{M} 1$ and $\mathrm{M} 2$ counts (q1, q3) were $2.3(0.9,12.2)$ and $7.6(4.4,13.9)$, respectively. M1 macrophages were found statistically significantly less frequent in the glomeruli in comparison with M2 macrophages $(p<0.001, b=-0,289 \pm 0.053)$. Collected laboratory data included inflammatory markers and markers of kidney function. There was no significant correlation between M1 and M2 macrophage count and laboratory parameters. Four pathohistological classifications were used: ISKDC, Haas classification, Oxford classification, and SQC classification. Classification stages/total classification scores and all histological variables were evaluated for possible correlation with macrophage count. Statistically significant negative correlations were found between segmental glomerulosclerosis (Oxford classification) and M2 macrophages $(\mathrm{p}=0,001, \mathrm{~b}=-1,050 \pm 0,275)$. An indication of negative correlations was noted between M2 macrophages and segmental sclerosis, and M2 macrophages and adhesions (SQC classification).

Conclusion Glomeruli in HSPN showed predominant M2 polarization of macrophages. M2 macrophage infiltration of glomeruli was highlighted as a possible negative predictor for segmental glomerulosclerosis.

This project is funded by Croatian Science Foundation project PURPURAPREDICTORS IP-2019-04-8822 and University of Rijeka, Croatia grant No. Uni-ri-biomed-18-110. 